\title{
VARIAÇÃO DA ACELERAÇÃO DA GRAVIDADE COM A LATITUDE E ALTITUDE
}

Wilson Lopes

Departamento de Física - Universidade Guarulhos

Guarulhos - SP

\section{Resumo}

Propõe-se, neste trabalho, uma equação para o módulo do vetor aceleração da gravidade, variando com a latitude e altitude. Para essa finalidade, foram usados os seguintes valores para a aceleração da gravidade, ao nível do mar: no equador, $g_{0}=9,7803 \mathrm{~m} / \mathrm{s}^{2}$ $e$, na latitude de $45^{\circ}, g_{P}=9,8062 \mathrm{~m} / \mathrm{s}^{2}$. O perfil terrestre foi assumido como sendo um elipsóide de revolução, achatado nos pólos, e a aceleração da gravidade variando com a altitude, em relação ao nível do mar, foi considerada também dependente da latitude.

Palavras-chaves: Elipsóide de revolução; variação da aceleração da gravidade; latitude e altitude.

\begin{abstract}
The propose of this work is an equation for the module of the acceleration vector of the gravity, varying with the latitude and altitude. For this purpose, the following values of the gravity acceleration were used, at the sea level: in the equator, $g_{0}=9,7803 \mathrm{~m} / \mathrm{s}^{2}$, and in the latitude of $45^{\circ}, g_{P}=9,8062 \mathrm{~m} / \mathrm{s}^{2}$. The terrestrial profile were assumed as being a revolution ellipsoid, flattened in the poles, and the acceleration of the gravity varying with the altitude, at sea level, was considered dependent of the latitude too.
\end{abstract}

\footnotetext{
${ }^{+}$Variation of the gravity acceleration with the latitude and altitude

* Recebido: novembro de 2007.

Aceito: julho de 2008.
} 
Keywords: Revolution ellipsoid; acceleration variation of gravity; latitude and altitude.

\section{Introdução}

É notória a dificuldade de alguns alunos, que ingressam no curso superior, em admitir que a aceleração da gravidade não seja uma grandeza física constante. Afinal, é natural que, após um longo período de tempo (pelo menos três anos) usando $9,8 \mathrm{~m} / \mathrm{s}^{2}$ (ou seu valor aproximado para $10 \mathrm{~m} / \mathrm{s}^{2}$ ) ficasse consolidado, na mente do aluno, essa grandeza física como sendo "constante". Contudo, o vetor aceleração da gravidade varia em módulo, direção e sentido. Sugere-se, portanto, que o professor de Física, no curso secundário e mesmo no início do curso superior, discuta com seus alunos a respeito desse tema, mostrando aos mesmos que o peso de qualquer objeto é função do vetor aceleração da gravidade.

Para superar essas dificuldades, procura-se estabelecer, neste artigo, uma equação simples da variação da aceleração da gravidade com a latitude e altitude, que forneça valores compatíveis com a equação internacional, até o quinto algarismo significativo.

A Terra, devido ao seu movimento de rotação, com velocidade angular $\mathrm{w}=7,292 \times 10^{-5} \mathrm{rad} / \mathrm{s}$, é achatada nos pólos, assemelhando-se a um elipsóide de revolução, em que o raio equatorial $\mathrm{a}=6,378 \times 10^{6} \mathrm{~m}$ é maior que o polar $\mathrm{b}=6,357 \times 10^{6} \mathrm{~m}$. Seu volume é dado por: $\mathrm{V}=4 \pi \mathrm{a}^{2} \mathrm{~b} / 3=1,083 \times 10^{21} \mathrm{~m}^{3}$. Na suposição de uma Terra perfeitamente esférica, com o mesmo volume do elipsóide de revolução, seu raio assumiria o valor: $R=\left[3.1,083 \times 10^{21} /(4 \pi)\right]^{1 / 3}=6,371 \times 10^{6} \mathrm{~m}$ (STACEY, 1977).

Devido a essa velocidade angular e a esse achatamento, a aceleração da gravidade, ao nível do mar, aumenta ao percorrer-se um meridiano no sentido dos pólos, segundo a equação internacional:

$$
g(\lambda)=9,78032677\left(1+0,0052790414 \cdot \operatorname{sen}^{2} \lambda+0,0000232718 \cdot \operatorname{sen}^{4} \lambda\right)
$$

em que $\lambda$ representa a latitude medida em graus (MAROJA; VITURINO; PEREIRA, 2007).

Uma equação mais simples e que fornece valores, com cinco algarismos significativos, praticamente iguais à equação (1), para a aceleração da gravidade, variando com a latitude e ao nível do mar, é dada por:

$$
g_{\lambda}=g_{0}\left(1+\beta \operatorname{sen}^{2} \lambda\right)
$$


em que $\mathrm{g}_{0}=9,7803 \mathrm{~m} / \mathrm{s}^{2}$ representa um valor aproximado da aceleração da gravidade, no equador e ao nível do mar, obtido com a equação (1), e $\beta=5,300 \times 10^{-3}$ é um fator numérico que leva em conta a rotação terrestre, em torno de seu eixo, e o achatamento polar devido a essa rotação (COOK, 1969). Se levássemos em conta somente a aceleração centrípeta, esse coeficiente numérico seria: $\beta \approx 3,388 \times 10^{-3}$ (RESNICK; HALLIDAY, 1970). Entretanto, o valor desse coeficiente numérico é 1,564 vezes maior, ou seja, a contribuição do achatamento da Terra, para o coeficiente numérico é, aproximadamente, $1,912 \times 10^{-3}$.

Tabela 1: Estes valores da aceleração da gravidade, ao nível do mar e variando com a latitude, foram gerados com as equações (1) e (2).

\begin{tabular}{|c|cccccccccc|}
\hline Equação & \multicolumn{10}{|c|}{ Latitude em graus } \\
& 0 & 10 & 20 & 30 & 40 & 50 & 60 & 70 & 80 & 90 \\
\hline$[1]$ & 9,7803 & 9,7819 & 9,7864 & 9,7932 & 9,8017 & 9,8107 & 9,8192 & 9,8261 & 9,8306 & 9,8322 \\
{$[2]$} & 9,7803 & 9,7819 & 9,7864 & 9,7933 & 9,8017 & 9,8107 & 9,8192 & 9,8261 & 9,8306 & 9,8321 \\
\hline
\end{tabular}

\section{Variação da aceleração da gravidade com a latitude e altitude} ção :

Com relação à altitude, a aceleração da gravidade varia segundo a equa-

$$
\frac{\Delta g_{z}}{g_{\lambda}} \approx-\frac{2 z}{R}
$$

Substituindo-se, na equação (3), $\mathrm{g}_{\lambda}$ definido pela equação (2), obtém-se:

$$
\Delta g_{z} \approx-\frac{2 g_{0}\left(1+\beta \operatorname{sen}^{2} \lambda\right) \cdot z}{R}
$$

em que z é altitude, localizada a uma determinada latitude $\lambda$, e $\mathrm{R}=6,371 \times 10^{6} \mathrm{~m}$ é o raio da Terra, suposta esférica, com o mesmo volume do elipsóide de revolução que, nesse caso, melhor representa a figura terrestre.

A variação da aceleração da gravidade, em função da latitude e altitude, pode ser definida por:

\footnotetext{
${ }^{1}$ Halliday e Resnick (1970, p. 417-418) apresentam uma equação de variação da aceleração da gravidade com a altitude, um pouco diferente: $\mathrm{dg} / \mathrm{g}=-2 \mathrm{dr} / \mathrm{r}$. No nosso caso, na equação (3), estamos supondo $z$ muito menor que o raio terrestre $(z<<R)$, como se fosse o infinitésimo dr.
} 


$$
\begin{aligned}
& g_{\lambda, z} \approx g_{\lambda}+\Delta g_{z} \\
& \approx g_{0}\left(1+\beta \operatorname{sen}^{2} \lambda\right)-\frac{2 g_{0}\left(1+\operatorname{sen}^{2} \lambda\right) z}{R} \\
& \approx g_{0}\left(1+\beta \operatorname{sen}^{2} \lambda\right)(1-2 z / R)
\end{aligned}
$$

A equação (5) fornece a aceleração da gravidade, em função da aceleração da gravidade no equador e ao nível do mar, da latitude e altitude (LOPES, 1999).

Na equação (2), pode-se assumir $g_{\lambda}=g_{45^{\circ}}=9,8062 \mathrm{~m} / \mathrm{s}^{2}$ que representa a aceleração da gravidade padrão, ao nível do mar e na latitude $\lambda=45^{\circ}$, que será indicada por $g_{P}$. Portanto:

$$
g_{0}=\frac{g_{P}}{1+\beta \cdot \operatorname{sen}^{2} 45^{0}}=\frac{g_{P}}{1+\beta / 2}
$$

Substituindo-se (6) em (5), obtém-se:

$$
g_{\lambda, z} \approx \frac{g_{p}}{1+\beta / 2}\left(1+\beta \operatorname{sen}^{2} \lambda\right)(1-2 z / R)
$$

A equação (7), da mesma maneira que a equação (5), fornece os valores da aceleração da gravidade, em função da gravidade considerada padrão, $\mathrm{g}_{\mathrm{P}}=$ $9,8062 \mathrm{~m} / \mathrm{s}^{2}$, em função da latitude e altitude ${ }^{2}$.

Tabela 2: Variação da aceleração da gravidade com a altitude, na latitude de $45^{\circ}$.

\begin{tabular}{|c|c|c|}
\hline $\begin{array}{c}\text { Altitude } \\
(\mathrm{km})\end{array}$ & $\mathrm{g}_{\lambda, \mathrm{z}}^{*}\left(\mathrm{~m} / \mathrm{s}^{2}\right)$ & $\mathrm{g}_{\lambda, \mathrm{z}}\left(\mathrm{m} / \mathrm{s}^{2}\right)$ \\
\hline 0 & 9,806 & 9,8062 \\
1 & 9,803 & 9,8031 \\
4 & 9,794 & 9,7939 \\
8 & 9,782 & 9,7816 \\
16 & 9,757 & 9,7570 \\
32 & 9,708 & 9,7077 \\
100 & 9,598 & 9,4984 \\
& & \\
\hline
\end{tabular}

${ }^{2}$ Devido à aproximação introduzida pela equação (3), não convém calcular a aceleração da gravidade, com as equações (5) ou (7), para valores da altitude superiores a $32 \mathrm{~km}$, conforme a Tabela 2. Os valores da segunda coluna, da Tabela 2, são encontrados no livro de Resnick e Halliday (1970) e os da terceira coluna foram obtidos com o auxílio das equações (5) ou (7). 


\section{Conclusão}

Existem balanças eletrônicas, nos laboratórios de Física, que medem massa até centésimos de grama. Como exemplo, suponhamos que essa balança de precisão mediu a massa de um objeto $\mathrm{m}=11,91 \mathrm{~g}$, com quatro algarismos significativos, num laboratório localizado na cidade de São Paulo $\left(\lambda \approx-22,55^{\circ}\right.$ de latitude; $\mathrm{z}=760 \mathrm{~m}$ de altitude). Não seria conveniente determinar o peso do objeto, usando-se o valor aproximado da aceleração da gravidade $\mathrm{g}=10 \mathrm{~m} / \mathrm{s}^{2}\left(\mathrm{~g}=1,0 \times 10^{3}\right.$ $\mathrm{cm} / \mathrm{s}^{2}$ ), encontrando-se $\mathrm{P}=\mathrm{mg}=11,91.1,0 \times 10^{3}=1,2 \times 10^{4}$ dina, ou $1,1 \times 10^{4}$ dina (com o primeiro ou segundo resultado para o módulo da força peso, o segundo algarismo é duvidoso). Seria melhor, através das equações (5) ou (7), avaliar-se a aceleração da gravidade local, $\mathrm{g}=978,56 \mathrm{~cm} / \mathrm{s}^{2}\left(\mathrm{~g}=9,7856 \mathrm{~m} / \mathrm{s}^{2}\right)$ e multiplicá-la pela massa, a saber: $\mathrm{P}=\mathrm{m} \mathrm{g}=11,91.978,56=1,1655 \times 10^{4}$ dina. Se em todos os problemas de Física que, por comodidade, tornou-se um hábito, até mesmo nas universidades, de se considerar a aceleração da gravidade $10 \mathrm{~m} / \mathrm{s}^{2}$ ou $9,8 \mathrm{~m} / \mathrm{s}^{2}$, em relação à força peso do exemplo exposto acima, não haveria a necessidade de uma balança tão precisa.

Qual é a diferença de peso de uma pessoa, de massa $\mathrm{m}=80,00 \mathrm{~kg}$, ao se deslocar, ao nível do mar, do equador até o pólo? Essa pergunta, de acordo com este texto, responde-se da seguinte maneira:

$$
\Delta \mathrm{P}=80,00(9,8321-9,7803)=80,00.0,0518=4,144 \mathrm{~N} \text { (verifique as a- }
$$
celerações da gravidade no equador e no pólo na Tabela 1$)$.

Da mesma maneira, qual é a variação do peso da mesma pessoa de $80,00 \mathrm{~kg}$, ao deslocar-se da cidade de Santos (latitude $-23,96^{\circ}$; altitude 2,00 m), $\mathrm{g}=9,7888 \mathrm{~m} / \mathrm{s}^{2}$, à cidade de Campos do Jordão (latitude -22,74 ${ }^{0}$; altitude $1628 \mathrm{~m}$ ), $\mathrm{g}=9,7830 \mathrm{~m} / \mathrm{s}^{2}$ ?

A variação no peso da pessoa é dada por:

$\Delta \mathrm{P}=80,00(9,7888-9,7830)=80,00.0,0058=0,464 \mathrm{~N}$ (calculem as acelerações da gravidade com as equações (5) ou (7)).

Medidas experimentais da aceleração da gravidade, com cinco ou mais algarismos significativos, comparadas com os valores teóricos da aceleração da gravidade, variando em função da latitude e altitude, poderiam revelar algumas anomalias dessa grandeza física na crosta terrestre. Encontrando-se, experimentalmente, para a aceleração da gravidade, numa certa localidade, um valor sensivelmente menor que o valor indicado pelas equações (5) ou (7), poderia revelar no subsolo uma jazida petrolífera; encontrando-se um valor sensivelmente maior, poderia revelar, no subsolo, a presença de minerais metálicos, com uma densidade 
superior à da crosta terrestre (a Tabela 3 apresenta valores da aceleração da gravidade, em função da latitude e altitude, obtidos com a equação (5)).

A maioria dos livros de Física, do curso secundário (e até mesmo do superior) apresenta, para facilitar os cálculos, a aceleração da gravidade: 10, 9,8 e $9,81 \mathrm{~m} / \mathrm{s}^{2}$ (este último valor raramente é encontrado). Concordo, plenamente, que o valor mais usado, $g=10 \mathrm{~m} / \mathrm{s}^{2}$, representa uma grande facilidade no desenvolvimento dos cálculos para se chegar à solução de um problema de Física. Contudo, essa insistência no valor de $10 \mathrm{~m} / \mathrm{s}^{2}$ leva a maioria dos alunos a pensar que se trata de uma grandeza física constante. Para se contornar esse problema, desde o curso secundário, os professores deveriam discutir com seus alunos sobre as variações do módulo da aceleração da gravidade com a latitude e altitude. Para mostrar que se trata de um vetor variável, seria muito fácil: bastaria desenhar a Terra e representálo perpendicularmente à superfície terrestre no equador e nos pólos, mostrando que a direção e o sentido do vetor são variáveis. Após esses cuidados, mesmo que o aluno usasse o valor $10 \mathrm{~m} / \mathrm{s}^{2}$, estaria ciente de que se trata de uma grandeza física variável, muito importante quando obtida experimentalmente com um número maior de algarismos significativos.

\section{Bibliografia}

COOK, A. H. Gravity and the Earth. London: Wykeham Publcations LTD, $1969.95 \mathrm{p}$.

LOPES, W. Uma equação barométrica coerente com a equação de Laplace. Revista Brasileira de Ensino de Física. v. 21, n. 4, p. 524, 1999.

MAROJA, A. M.; VITURINO, M. F. C.; PEREIRA, J. de S. Medida da Aceleração da Gravidade. Disponível em:

$<$ www.sbf1.sbfísica.org.br/eventos/snef/xvi/cd/resumos/T0297.pdf>. Acesso em: 16 nov. 2007.

RESNICK, R; HALLIDAY, D. Física. Parte I. Tradução: Euclydes Cavallari e Bento Afini Jr. Rio de Janeiro: Ao Livro Técnico S. A., 1970. 739 p.

STACEY, F. D. Physics of the Earth. 2. ed. New York: John Willey \& Sons, 1977. $414 \mathrm{p}$. 


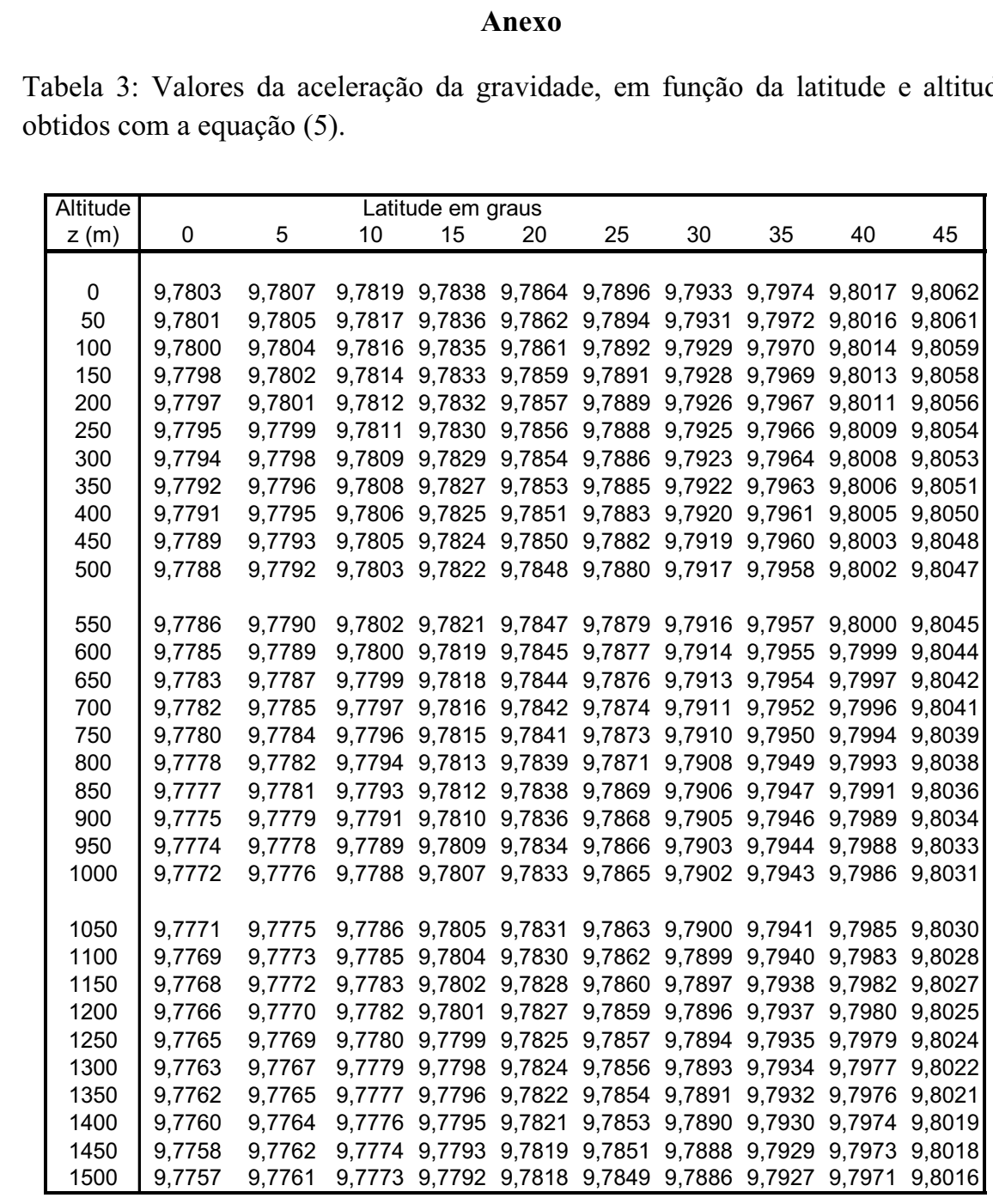


Tabela 3 (continuação): Valores da aceleração da gravidade, em função da latitude e altitude, obtidos com a equação (5).

\begin{tabular}{|c|c|c|c|c|c|c|c|c|c|}
\hline \multirow{2}{*}{$\begin{array}{c}\text { Altitude } \\
\mathrm{z}(\mathrm{m})\end{array}$} & \multicolumn{9}{|c|}{ Latitude em graus } \\
\hline & 50 & 55 & 60 & 65 & 70 & 75 & 80 & 85 & 90 \\
\hline 0 & 9,8107 & 9,8151 & 9,8192 & 9,8229 & 9,8261 & 9,8287 & 9,8306 & 9,8317 & 9,8321 \\
\hline 50 & 9,8106 & 9,8149 & 9,8190 & 9,8227 & 9,8259 & 9,8285 & 9,8304 & 9,8316 & 9,8320 \\
\hline 100 & 9,8104 & 9,8148 & 9,8189 & 9,8226 & 9,8258 & 9,8284 & 9,8303 & 9,8314 & 9,8318 \\
\hline 150 & 9,8103 & 9,8146 & 9,8187 & 9,8224 & 9,8256 & 9,8282 & 9,8301 & 9,8313 & 9,8317 \\
\hline 200 & 9,8101 & 9,8145 & 9,8186 & 9,8223 & 9,8255 & 9,8280 & 9,8300 & 9,8311 & 9,8315 \\
\hline 250 & 9,8099 & 9,8143 & 9,8184 & 9,8221 & 9,8253 & 9,8279 & 9,8298 & 9,8310 & 9,8314 \\
\hline 300 & 9,8098 & 9,8142 & 9,8182 & 9,8219 & 9,8251 & 9,8277 & 9,8296 & 9,8308 & 9,8312 \\
\hline 350 & 9,8096 & 9,8140 & 9,8181 & 9,8218 & 9,8250 & 9,8276 & 9,8295 & 9,8307 & 9,8311 \\
\hline 400 & 9,8095 & 9,8138 & 9,8179 & 9,8216 & 9,8248 & 9,8274 & 9,8293 & 9,8305 & 9,8309 \\
\hline 450 & 9,8093 & 9,8137 & 9,8178 & 9,8215 & 9,8247 & 9,8273 & 9,8292 & 9,8304 & 9,8307 \\
\hline 500 & 9,8092 & 9,8135 & 9,8176 & 9,8213 & 9,8245 & 9,8271 & 9,8290 & 9,8302 & 9,8306 \\
\hline 550 & 9,8090 & 9,8134 & 9,8175 & 9,8212 & 9,8244 & 9,8270 & 9,8289 & 9,8300 & 9,8304 \\
\hline 600 & 9,8089 & 9,8132 & 9,8173 & 9,8210 & 9,8242 & 9,8268 & 9,8287 & 9,8299 & 9,8303 \\
\hline 650 & 9,8087 & 9,8131 & 9,8172 & 9,8209 & 9,8241 & 9,8267 & 9,8286 & 9,8297 & 9,8301 \\
\hline 700 & 9,8086 & 9,8129 & 9,8170 & 9,8207 & 9,8239 & 9,8265 & 9,8284 & 9,8296 & 9,8300 \\
\hline 750 & 9,8084 & 9,8128 & 9,8169 & 9,8206 & 9,8238 & 9,8263 & 9,8283 & 9,8294 & 9,8298 \\
\hline 800 & 9,8083 & 9,8126 & 9,8167 & 9,8204 & 9,8236 & 9,8262 & 9,8281 & 9,8293 & 9,8297 \\
\hline 850 & 9,8081 & 9,8125 & 9,8166 & 9,8203 & 9,8234 & 9,8260 & 9,8279 & 9,8291 & 9,8295 \\
\hline 900 & 9,8079 & 9,8123 & 9,8164 & 9,8201 & 9,8233 & 9,8259 & 9,8278 & 9,8290 & 9,8294 \\
\hline 950 & 9,8078 & 9,8122 & 9,8162 & 9,8199 & 9,8231 & 9,8257 & 9,8276 & 9,8288 & 9,8292 \\
\hline 1000 & 9,8076 & 9,8120 & 9,8161 & 9,8198 & 9,8230 & 9,8256 & 9,8275 & 9,8287 & 9,8290 \\
\hline 1050 & 9,8075 & 9,8118 & 9,8159 & 9,8196 & 9,8228 & 9,8254 & 9,8273 & 9,8285 & 9,8289 \\
\hline 1100 & 9,8073 & 9,8117 & 9,8158 & 9,8195 & 9,8227 & 9,8253 & 9,8272 & 9,8283 & 9,8287 \\
\hline 1150 & 9,8072 & 9,8115 & 9,8156 & 9,8193 & 9,8225 & 9,8251 & 9,8270 & 9,8282 & 9,8286 \\
\hline 1200 & 9,8070 & 9,8114 & 9,8155 & 9,8192 & 9,8224 & 9,8250 & 9,8269 & 9,8280 & 9,8284 \\
\hline 1250 & 9,8069 & 9,8112 & 9,8153 & 9,8190 & 9,8222 & 9,8248 & 9,8267 & 9,8279 & 9,8283 \\
\hline 1300 & 9,8067 & 9,8111 & 9,8152 & 9,8189 & 9,8221 & 9,8246 & 9,8266 & 9,8277 & 9,8281 \\
\hline 1350 & 9,8066 & 9,8109 & 9,8150 & 9,8187 & 9,8219 & 9,8245 & 9,8264 & 9,8276 & 9,8280 \\
\hline 1400 & 9,8064 & 9,8108 & 9,8149 & 9,8186 & 9,8218 & 9,8243 & 9,8263 & 9,8274 & 9,8278 \\
\hline 1450 & 9,8062 & 9,8106 & 9,8147 & 9,8184 & 9,8216 & 9,8242 & 9,8261 & 9,8273 & 9,8277 \\
\hline
\end{tabular}

